\title{
The evolution of disability after ischemic stroke depending on the circadian variation of stroke onset
}

Dana Marieta Fodor, Ioana Cristina Stănescu, Lăcrămioara Perju-Dumbravă

Corresponding author: : Dana Marieta Fodor, E-mail address: fodordana@yahoo.com

\begin{abstract}
Introduction. The circadian variation of ischemic stroke onset is known, but its impact on recovery prognosis has been less studied. Materials and method. The study included 32 patients with ischemic stroke, admitted to Neurology Departments I and II of the Rehabilitation Hospital in Cluj-Napoca between 1 June 2008 and 31 December 2009 and followed up for 2 years, during 5 successive admissions. The diagnosis of ischemic stroke was defined according to updated World Health Organization criteria. The onset time of ischemic stroke was assigned to one of the following six-hour intervals: 00.01-06.00 (night), 06.01-12.00 (morning), 12.01-18.00 (afternoon) and 18.01-24.00 (evening). For each patient we recorded demographic data and the values of ADL and IADL scales on the occasion of each assessment. Statistical analysis was performed using Excel Microsoft, descriptive and one-way ANOVA test. Results and conclusions. Our study confirms the incidence pattern of ischemic stroke, with a morning peak, which is more obvious in the case of the male sex and patients aged less than 65 years. Patients with nocturnal stroke onset have a less favorable functional evolution during the second year after ischemic stroke.
\end{abstract}

Key words: ischemic stroke occurrence, circadian variation, post-stroke functional status,

\section{Introduction}

The presence of a circadian variation in ischemic stroke onset has been described in the literature, with some differences between different authors. However, most of the data support an incidence peak in the morning and a second one, inconsistently described, in the afternoon [1-5].

The degree of disability or functional status after stroke can be assessed using several scales. One of them is the ADL (Activities of Daily Living) Scale, complemented by the IADL (Instrumental Activities of Daily Living) Scale. The first evaluates an individual's ability to perform daily activities required for personal needs ( 6 items with a maximum score of 6 points: dressing, personal hygiene, toileting, continence, walking/transfers, feeding) and the second measures the degree of independence within the community, including more complex activities (financial management, telephone use, house cleaning, meal preparation, public transportation use, shopping, laundry, selfmedication). Changes occurring in IADL suggest mild cognitive decline, while ADL changes suggest moderate/advanced cognitive decline, along with other neurological deficits that create disability as a consequence of cerebrovascular pathology [6-9]. The impact of the circadian variation of ischemic stroke onset on basic (ADL) and instrumental (IADL) functional status and, moreover, its dynamics have been less studied [10-12].

\section{Materials and method}

Our results are based on a prospective cohort study including 32 patients with recent ischemic stroke (over the past 6 months), admitted to Neurology Departments I and II of the Rehabilitation Hospital in Cluj-Napoca, between 1 June 2008 and 31 December 2009. The diagnosis of ischemic stroke was confirmed by neurologic examination and neuroimaging, being defined according to updated World Health Organization criteria. For each patient, we recorded demographic data and the onset time (determined or approximated by history taking) was assigned to one of the following six-hour intervals: 00.01-06.00 (night), 06.01-12.00 (morning), 12.0118.00 (afternoon) and 18.01-24.00 (evening). For cases in which symptoms were first recognized at wake-up time, this was considered the time of onset (3 cases).

The 63 patients were assessed on the occasion of 5 visits: at the first examination (time 0), at 1 month, 6 months, 12 months and 24 months. Four of these patients missed one visit (3 patients missed the second examination - " 1 " and 1 patient missed the last examination - "24"). On the occasion of each visit, the patients were evaluated based on the two scales: ADL (score 1-6) and IADL (score 1-8).

Statistical analysis was performed using Excel Microsoft software. In descriptive statistical calculations, categorical data were presented as diagrams, absolute and relative frequencies, and 
continuous variables were summarized using synthetic centrality, dispersion and location indicators, or frequency histograms and linear diagrams.

\section{Results}

In our study, ischemic stroke onset had the highest frequency in the 6-12 interval, followed by the 12-18 interval (Figs. 1 and 2), while the lowest frequency was found in the 0-6 interval. Frequency depending on the patients' sex is shown in Figure 3. Depending on age, the patients were divided into two groups ( $<65$ years, $\geq 65$ years) - Figure 4 . Figures 5 and 6 represent the evolution of the arithmetic mean of ADL and IADL scores across the 4 time intervals of the day over 2 years ( 5 evaluations).

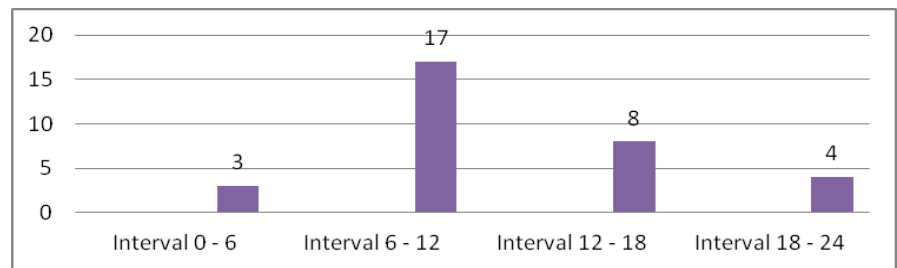

Fig. 1: Circadian variation of ischemic stroke onset

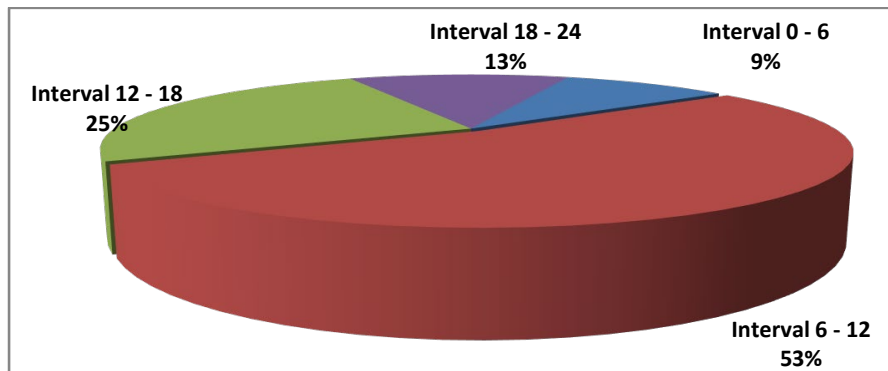

Fig. 2: Percentage representation of ischemic stroke onset across the 4 time intervals of the day

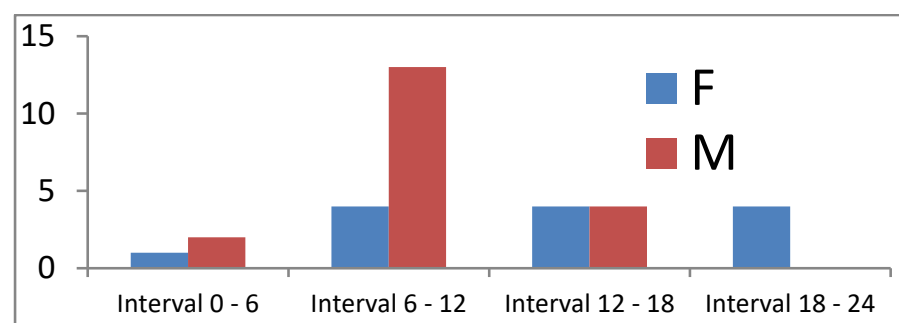

Fig. 3: Circadian variation of stroke onset depending on sex (Ffemale, M-male)

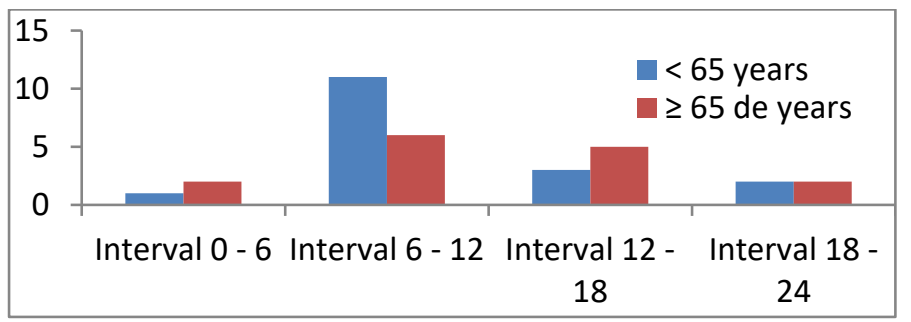

Fig. 4: Circadian variation of stroke onset depending on age

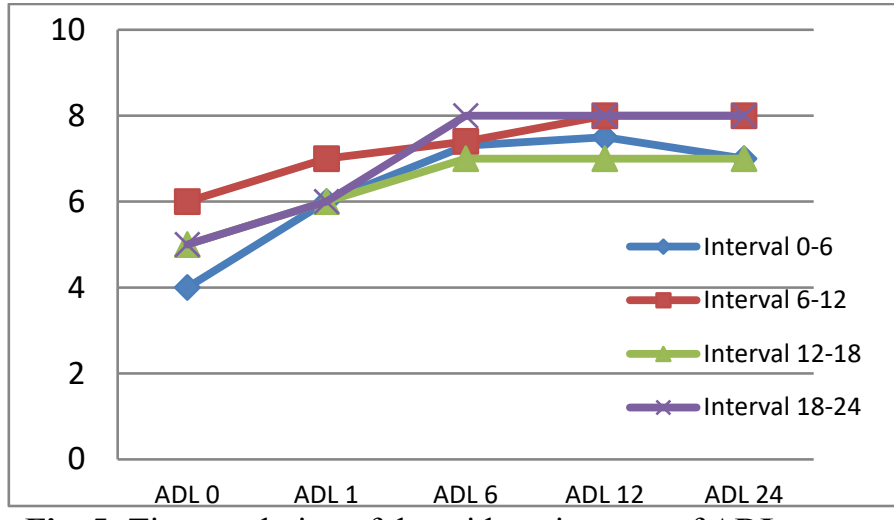

Fig. 5: Time evolution of the arithmetic mean of ADL scores across the 5 evaluations over 2 years, for the 4 time intervals of the day

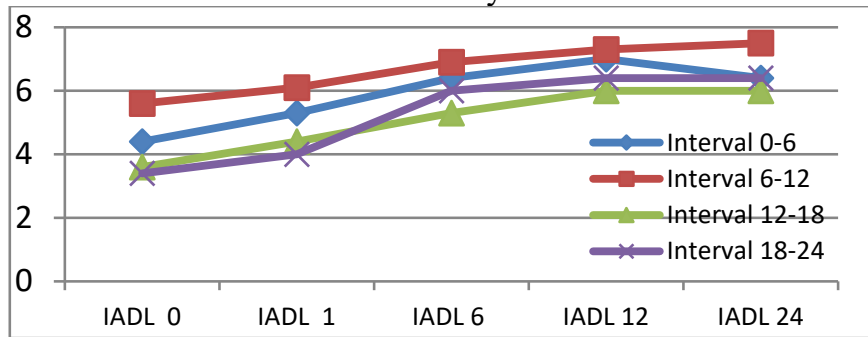

Fig. 6: Time evolution of the arithmetic mean of IADL scores across the 5 evaluations over 2 years, for the 4 time intervals of

\section{Discussions} the day

The investigation of the circadian variation of ischemic stroke onset in the study group revealed an incidence peak in the morning interval, 06.01-12.00 $(p=0.06)$, in accordance with the reported literature data [1-5]. Although most of the data are derived from retrospective studies on large numbers of patients, there are also some prospective studies that report the same circadian variation of stroke onset $[13,14]$. In our study, the morning peak is more obvious in the case of the male sex and patients aged less than 65 years $(\mathrm{p}=0.046)$.

Descriptive statistics shows, following analysis of diagrams for the dynamics of ADL and IADL scores across the 5 measurements over 2 years, that the highest score for the patients included in the study was obtained for the 6-12 interval. The most affected ADL score was that of patients with stroke onset in the 0-6 interval, and the lowest IADL score was found for patients suffering stroke in the 12-18 and 18-24 intervals. Both the mean ADL and IADL score of patients with stroke onset in the 0-6 interval ( 3 cases) had an initial favorable evolution for up to 12 months, after which a decline was detected at 24 months. For the other intervals $(6-12,12-18,18-24)$, the evolution of the mean scores improved during the first 12 months and was maintained relatively unchanged 
over the following year (except for the 6-12 interval for IADL), suggesting that the degree of disability and instrumental independence have the greatest improvement within one year of ischemic stroke, regardless of the onset time. The ADL and IADL scores had a relatively parallel evolution in the patients of our study.

Although Anova statistical analysis for the comparison of the mean ADL and IADL scores in the four groups of patients corresponding to the 4 intervals of the day, on the occasion of the 5 evaluations, evidenced no statistically significant differences (most probably due to the small number of patients in the study), descriptive analysis supports a less favorable functional evolution during the second year after ischemic stroke for onset in the nocturnal interval (0-6).

In the literature there are few data related to the evolution of disability scores (modified Rankin score - mRS) depending on the circadian interval of stroke onset, assessments being performed only at the initial admission, not during evolution over a longer patient follow-up period. The results somewhat overlap, describing the best functional score during evolution from admission to discharge for ischemic stroke patients with onset in the 04.01-08.00 interval and the lowest functional score in the 20.01-24.00 interval [5, 12].

\section{Conclusions}

The study confirms the circadian variation pattern of ischemic stroke, with a peak of occurrence in the morning hours and the lowest frequency in the nocturnal interval.

The evolution of ADL and IADL scores across the 4 time intervals of the day over 2 years is similar in our study group, with the greatest benefit during the first year after stroke, and a subsequently unchanged evolution, excepting onset in the nocturnal interval (0-6), for which a slight decline during the second year after stroke is described.

Information related to post-ischemic stroke evolution in terms of functional and cognitive recovery, taking into consideration the circadian variation of stroke onset, deserves to be extended in larger studies, because it can be important for estimating recovery prognosis and the resources involved in the rehabilitation process.

\section{References}

1. Manfredini R, Boari B, Smolensky MH, Salmi R, la Cecilia O, Maria Malagoni A, et al. Circadian Variation in Stroke Onset: Identical Temporal Pattern in Ischemic and Hemorrhagic Events. Chronobiology International. 2009;22(3):417-53.

2. Turin TC, Kita Y, Rumana N, Nakamura Y, Takashima N, Ichikawa $\mathrm{M}$, et al. Is there any circadian variation consequence on acute case fatality of stroke? Takashima Stroke Registry, Japan (19902003). Acta Neurol Scand. 2012;125(3):206-12.

3. Fodor DM G-ND, Perju-Dumbrava L. Circadian patterns of ischemic stroke onset. HVM Bioflux. 2014;6(3):132-9.

4. Fodor DM, Babiciu I, Perju-Dumbrava L. Circadian Variation of Stroke Onset: A Hospital-Based Study. Clujul Med. 2014;87(4):242-9.

5. Ripamonti L, Riva R, Maioli F, Zenesini C, Procaccianti G. Daily Variation in the Occurrence of Different Subtypes of Stroke. Stroke Research and Treatment. 2017;2017:9091250.

6. Mlinac ME, Feng MC. Assessment of Activities of Daily Living, Self-Care, and Independence. Archives of Clinical Neuropsychology. 2016;31(6):506-16.

7. Blomgren C, Jood K, Jern C, Holmegaard L, Redfors $\mathrm{P}$, Blomstrand $\mathrm{C}$, et al. Long-term performance of instrumental activities of daily living (IADL) in young and middle-aged stroke survivors: Results from SAHLSIS outcome. Scandinavian Journal of Occupational Therapy. 2018;25(2):119-26.

8. Stanescu I, Dogaru G. Brain Natriuretic Peptide (Bnp): Biomarker for Risk Stratification and Functional Recovery Prediction in Ischemic Stroke. Balneo Research Journal. 2015;6(1):40-4.

9. Harrison JK, McArthur KS, Quinn TJ. Assessment scales in stroke: clinimetric and clinical considerations. Clinical Interventions in Aging. 2013;8:201-11.

10. Ghandehari K. Challenging comparison of stroke scales. Journal of Research in Medical Sciences: the Official Journal of Isfahan University of Medical Sciences. 2013;18(10):906-10.

11. Jimenez-Conde J, Ois A, Rodriguez-Campello A, Gomis M, Roquer J. Does sleep protect against ischemic stroke? Less frequent ischemic strokes but more severe ones. Journal of Neurology. 2007;254(6):782-8.

12. Liou LM, Lin HF, Tsai CL, Lin RT, Lai CL. Timing of stroke onset determines discharge-functional status but not stroke severity: a hospital-based study. The Kaohsiung Journal of Medical Sciences. 2013;29(1):32-6.

13. Gupta A, Shetty H. Circadian variation in stroke - a prospective hospital-based study. Int J Clin Pract. 2005;59(11):1272-5

14. Uddin MS, Hoque MI, Uddin MK, Kamol SA, Chowdhury RH. Circadian rhythm of onset of stroke - in 50 cases of ischemic stroke. Mymensingh Medical Journal: MMJ. 2015;24(1):121-6. 